\title{
ON THE ZEROS OF POLYNOMIALS OVER DIVISION RINGS
}

\author{
BY \\ B. GORDON( $\left.{ }^{1}\right)$ AND T. S. MOTZKIN $\left({ }^{2}\right)$
}

1. Introduction. Let $f(x)$ be a polynomial of degree $n$ with coefficients in the center $K$ of a division ring $D$. Herstein [1] has shown that the number of zeros of $f(x)$ in $D$ is either $\leqq n$ or infinite. In this paper we investigate the situation for polynomials whose coefficients are in $D$, but not necessarily in $K$. Here one must distinguish between two types of polynomials, which we call left and general.

A left polynomial is an expression of the form $f(x)=a_{0} x^{n}+a_{1} x^{n-1}+$ $\cdots+a_{n}$, where $a_{k} \in D(k=0, \cdots, n)$. Equality of two such polynomials is defined in the usual way. If $a_{0} \neq 0, n$ is called the degree of $f(x)$. If $c \in D$, we define $f(c)=a_{0} c^{n}+a_{1} c^{n-1}+\cdots+a_{n} ;$ if $f(c)=0, c$ is called a zero or root of $f(x)$. In $\$ 2$ we prove that the number of distinct zeros of a left polynomial of degree $n$ is either $\leqq n$ or infinite. This includes in particular a new proof of Herstein's result, avoiding the use of the CartanBrauer-Hua theorem.

Left polynomials can be added in the obvious way, and multiplied according to the rule $\left(a_{0} x^{m}+\cdots+a_{m}\right)\left(b_{0} x^{n}+\cdots+b_{n}\right)=c_{0} x^{m+n}+\cdots$ $+c_{m+n}$, where $c_{k}=\sum_{i+j=k} a_{i} b_{j}$; they then form a ring $D_{L}[x]$. However, the specialization maps $f(x) \rightarrow f(c)$ of $D_{L}[x]$ onto $D$ are not homomorphisms if $c \notin K$. To overcome this difficulty we are led to introduce general polynomials. Roughly speaking, a general polynomial is a sum of terms of the form $a_{0} x a_{1} x \ldots a_{k-1} x a_{k}$, where $a_{0}, \cdots, a_{k} \in D$. But there are certain identifications which must be made in order to obtain the various distributive laws, and to guarantee that $c x=x c$ for $c \in K$; therefore we now give a more careful description. Consider first the set $S$ of all finite sequences $\left(a_{0}, a_{1}, \cdots, a_{k}\right)$, where $a_{i} \in D$. It is easily seen that $S$ forms a semigroup under the product

$$
\left(a_{0}, a_{1}, \cdots, a_{k}\right)\left(b_{0}, b_{1}, \cdots, b_{l}\right)=\left(a_{0}, a_{1}, \cdots, a_{k-1}, a_{k} b_{0}, b_{1}, \cdots, b_{l}\right) .
$$

Let $R$ be the semigroup ring of $S$, and let $A_{i k}$ (where $0 \leqq i \leqq k$ ) be the set of all elements in $R$ of the form $\left(a_{0}, \cdots, a_{i}+b_{i}, \cdots, a_{k}\right)-\left(a_{0}, \cdots, a_{i}, \cdots, a_{k}\right)$ $-\left(a_{0}, \cdots, b_{i}, \cdots, a_{k}\right)$. Let $B_{k}$ be the set of all elements of $R$ of the form

Presented to the Society, August 25, 1964 under the title The number of zeros of a polynomial in a division ring; received by the editors May 15, 1964.

(1) Alfred P. Sloan Fellow.

( $\left.{ }^{2}\right)$ National Science Foundation Grant GP 2480. 
$\left(a_{0}, a_{1}, \cdots, a_{k}\right)-\left(c_{0} a_{0}, c_{1} a_{1}, \cdots, c_{k} a_{k}\right)$, where $c_{0}, \cdots, c_{k} \in K$, and $c_{0} c_{1} \cdots c_{k}=1$. We consider the quotient ring $R / a$, where $a$ is the ideal generated by

$$
\bigcup_{k=0}^{\infty}\left(\bigcup_{i=0}^{k} A_{i k} \cup B_{k}\right) \text {. }
$$

Each element $\left(a_{0}\right)$ constitutes a residue class $\bmod a$, and these classes form a subring $D^{\prime}$ of $R /$ a which is isomorphic to $D$. We now identify $D^{\prime}$ with $D$, and write $a_{0}$ instead of $\left(a_{0}\right)$. Let $x$ denote the residue class of $(1,1) \bmod a$; then it is easily verified that

$$
\left(a_{0}, a_{1}, \cdots, a_{k}\right) \equiv a_{0} x a_{1} x \cdots a_{k-1} x a_{k} \quad(\bmod \mathfrak{a}) .
$$

The elements of $R / a$ of the form $a_{0} x a_{1} x \ldots a_{k-1} x a_{k}$ are called general monomials, and denoted by symbols $M(x), M_{v}(x)$, etc. If $a_{0} a_{1} \cdots a_{k} \neq 0$, then $M(x)=a_{0} x \ldots x a_{k}$ is said to have degree $k$. Every element of $R / \mathfrak{a}$ can be represented as a sum $\sum_{\nu=1}^{m} M_{\nu}(x)$ of general monomials. Such elements are called general polynomials, and are denoted by symbols $f(x)$, $g(x)$, etc. It can be shown that every $f(x) \in R / \mathfrak{a}$ has a unique representation in the form $f(x)=\sum_{\nu=1}^{m} M_{\nu}(x)$ where $m$ is minimal. Then if $f(x)$ $\neq 0$, we define its degree to be $n=\max _{\nu} \operatorname{deg} M_{\nu}(x)$.

We are now justified in introducing the notation $D_{G}[x]$ for the ring $R / a$. There is a natural way of identifying $D_{L}[x]$ with a subset of $D_{G}[x]$, but this subset is not a subring of $D_{G}[x]$ unless $D=K$, in which case $D_{L}[x]$ $=D_{G}[x]=K[x]$. It is, however, always possible to map $D_{G}[x]$ homomorphically onto $D_{L}[x]$ by extending the map $a_{0} x a_{1} x \cdots x a_{k} \rightarrow a_{0} a_{1} \cdots a_{k} x^{k}$ to be additive.

In the construction of $D_{L}[x]$ and $D_{G}[x]$ we used only the fact that $D$ was a ring with identity; hence we can define $D_{L}\left[x_{1}, \cdots, x_{r}\right]$ and $D_{G}\left[x_{1}, \cdots, x_{r}\right]$ by induction.

If $c \in D$ and $M(x)=a_{0} x a_{1} \cdots x a_{k}$, put $M(c)=a_{0} c a_{1} \ldots c a_{k}$; it is clear from the definition of $a$ that $M(c)$ depends only on the residue class $\bmod a$ in which $\left(a_{0}, \cdots, a_{k}\right)$ lies, and is therefore well-defined. If $f(x)=\sum M_{\nu}(x)$, put $f(c)=\sum M_{\nu}(c)$; this is also well-defined. The specializations $f(x) \rightarrow f(c)$ are now homomorphisms of $D_{G}[x]$ onto $D$.

An element $c \in D$ is a zero or root of $f(x) \in D_{G}[x]$ if $f(c)=0$. Let $N(f)$ be the number of distinct zeros of $f(x)$. In $\$ 3$ we study $N(f)$ in the case where $K$ is infinite and $[D: K]=d<\infty$. We prove that if $h$ is any integer in the range $1 \leqq h \leqq n^{d}$, then there is a polynomial $f(x) \in D_{G}[x]$ of degree $n$ such that $N(f)=h$.

2. Left polynomials. Our first two theorems are essentially due to Richardson [3]; however his proofs are not quite correct, as pointed out by Rohrbach $[4]$. 
Theorem 1. An element $c \in D$ is a zero of a polynomial $f(x) \in D_{L}[x]$ if and only if there exists a $g(x) \in D_{L}[x]$ such that $f(x)=g(x)(x-c)$.

Proof. Let $f(x)=a_{0} x^{n}+a_{1} x^{n-1}+\cdots+a_{n}$. The theorem is trivial if $n=0$ or 1 , so we may suppose $n \geqq 2$. If $c$ is a root of $f(x)$, let

$$
\begin{aligned}
g(x)=a_{0} x^{n-1}+\left(a_{1}+a_{0} c\right) x^{n-2} & +\left(a_{2}+a_{1} c+a_{0} c^{2}\right) x^{n-3} \\
& +\cdots+\left(a_{n-1}+a_{n-2} c+\cdots+a_{0} c^{n-1}\right) .
\end{aligned}
$$

Then a simple calculation shows that $f(x)=g(x)(x-c)$.

Conversely, suppose that $f(x)=g(x)(x-c)$, where $g(x)=b_{0} x^{n-1}$ $+b_{1} x^{n-2}+\cdots+b_{n-1}$. Equating coefficients, we obtain

$$
\begin{aligned}
a_{0} & =b_{0}, \\
a_{1} & =b_{1}-b_{0} c, \\
a_{2} & =b_{2}-b_{1} c, \\
\vdots & \\
a_{n-1} & =b_{n-1}-b_{n-2} c, \\
a_{n} & =-b_{n-1} c .
\end{aligned}
$$

Multiplying the equation for $a_{i}$ on the right by $c^{n-i}$ and adding, we get $f(c)$ $=0$. This completes the proof.

We note that the existence of a factorization $f(x)=(x-c) h(x)$ neither implies nor is implied by $f(c)=0$.

Now let $D^{*}$ be the multiplicative group of nonzero elements of $D$. Two elements $a, b \in D$ are called conjugates if $a=t b t^{-1}$ for some $t \in D^{*}$. As usual this equivalence relation partitions $D$ into disjoint sets called conjugacy classes.

Theorem 2. If $f(x) \in D_{L}[x]$ has degree $n$, then at most $n$ conjugacy classes of $D$ contain roots of $f(x)$.

Proof. The proof is by induction on $n$. It is clear that a polynomial of degree zero has no roots, and a polynomial of degree one has exactly one root. Hence the theorem is true for $n<2$. Now suppose $n \geqq 2$, and assume that the theorem has already been proved for polynomials of degree $<n$. Suppose $f(x)=a_{0} x^{n}+a_{1} x^{n-1}+\cdots+a_{n}$ has $n+1$ distinct zeros $c_{0}, c_{1}$, $\cdots, c_{n}$. By Theorem 1 we have factorizations $f(x)=g_{i}(x)\left(x-c_{i}\right)(i=0, \cdots, n)$. Now assume $i>0$, and set $t_{i}=c_{i}-c_{0}$. Then $x-c_{0}=x-c_{i}+t_{i}$; hence

$$
\begin{aligned}
g_{i}(x)\left(x-c_{i}\right) & =g_{0}(x)\left(x-c_{0}\right) \\
& =g_{0}(x)\left(x-c_{i}\right)+g_{0}(x) t_{i} .
\end{aligned}
$$

Thus

$$
\begin{aligned}
g_{0}(x) & =\left[g_{i}(x)-g_{0}(x)\right]\left(x-c_{i}\right) t_{i}^{-1} \\
& =\left[g_{i}(x)-g_{0}(x)\right] t_{i}^{-1}\left(x-t_{i} c_{i} t_{i}^{-1}\right),
\end{aligned}
$$


remembering that $x t_{i}^{-1}=t_{i}^{-1} x$ in the ring $D_{L}[x]$. Another application of Theorem 1 now shows that $t_{i} c_{i} t_{i}^{-1}$ is a root of $g_{0}(x)(i=1, \cdots, n)$. Since $\operatorname{deg} g_{0}(x)<n$, the induction hypothesis implies that two of the elements $t_{i} c_{i} t_{i}^{-1}$ are conjugate (they may be equal). But if, say, $t_{1} c_{1} t_{1}^{-1}$ is conjugate to $t_{2} c_{2} t_{2}^{-1}$, then $c_{1}$ is conjugate to $c_{2}$, completing the induction.

THEOREM 3. If $D$ is a noncommutative division ring, then the centralizer $Z(c)$ of any element $c \in D$ is infinite.

Proof. We suppose $Z(c)$ is finite, and obtain a contradiction as follows. The center $K$ of $D$ is contained in $Z(c)$, so $K$ is a finite field; say $K=\operatorname{GF}(q)$. If $c \in K$, then $Z(c)=D$, which is infinite by Wedderburn's theorem. Hence $c \notin K$. Another application of Wedderburn's theorem shows that $Z(c)$ is a field, and hence $Z(c)=\mathrm{GF}\left(q^{f}\right)$, where $f>1$. The mapping $\mu: a \rightarrow a^{q}$ is an automorphism of $Z(c)$ with fixed field $K$. By a well-known theorem [2, p. 162], $\mu$ can be extended to an inner automorphism of $D$. Thus there is an element $t \in D$ such that $t a t^{-1}=a^{q}$ for all $a \in Z(c)$. In particular $t c t^{-1}=c^{q}$, and by iteration, $t^{f} c t^{-f}=c^{q^{f}}=c$. Hence $t^{f} \in Z(c)$, which implies that $t$ is of finite order. From these facts it follows easily that there are only a finite number of distinct elements of the form $\sum \lambda_{i, j} c^{i} t^{j} \quad\left(\lambda_{i, j} \in K\right)$ and that they form a subring $E \subset D$. The nonzero elements of $E$ form a finite semigroup $E^{*} \subset D^{*}$; hence $E^{*}$ is a group, and $E$ is a division ring. This contradicts Wedderburn's theorem, since $t c=c^{q} t \neq c t$.

Theorem 4. If a polynomial $f(x) \in D_{L}[x]$ has two distinct zeros in a conjugacy class of $D$, then it has infinitely many zeros in that class.

Proof. Suppose $c$ and $t c t^{-1} \neq c$ are zeros of $f(x)=a_{0} x^{n}+\cdots+a_{n}$. Consider the equation

$$
f\left(y c y^{-1}\right)=a_{0} y c^{n} y^{-1}+a_{1} y c^{n-1} y^{-1}+\cdots+a_{n}=0,
$$

where $y$ is the unknown. Except for the extraneous root 0 , this is equivalent to the equation

$$
a_{0} y c^{n}+a_{1} y c^{n-1}+\cdots+a_{n} y=0 .
$$

By hypothesis $y=1$ and $y=t$ are solutions of (2). Now (2) clearly has the following properties:

(i) If $y_{1}$ and $y_{2}$ are solutions, so is $y_{1}+y_{2}$.

(ii) If $y$ is a solution and $z \in Z(c)$, then $y z$ is a solution.

Combining these properties we see that $t+z$ is a solution of (2) for any $z \in Z(c)$. Moreover $t+z \neq 0$ since $t \in Z(c)$. Hence $t+z$ is a solution of (1), and so $(t+z) c(t+z)^{-1}$ is a zero of $f(x)$. To complete the proof we show that the elements $(t+z) c(t+z)^{-1}$ are all distinct and apply Theorem 3. Suppose that $\left(t+z_{1}\right) c\left(t+z_{1}\right)^{-1}=\left(t+z_{2}\right) c\left(t+z_{2}\right)^{-1}$, where $z_{1}, z_{2}$ $\in Z(c)$. Then $\left(t+z_{2}\right)^{-1}\left(t+z_{1}\right)$ commutes with $c$, so that $\left(t+z_{2}\right)^{-1}\left(t+z_{1}\right)$ $=z_{3}$ where $z_{3} \in Z(c)$. Thus $t+z_{1}=\left(t+z_{2}\right) z_{3}=t z_{3}+z_{2} z_{3}$. If $z_{3} \neq 1$, this 
implies that $t=\left(z_{2} z_{3}-z_{1}\right)\left(1-z_{3}\right)^{-1}$, which is in $Z(c)$ since $Z(c)$ is a division ring. This contradicts the fact that $t c t^{-1} \neq c$. Hence $z_{3}=1$, which means that $t+z_{1}=t+z_{2}$, or finally $z_{1}=z_{2}$.

Theorem 5. If $f(x) \in D_{L}[x]$ has degree $n$, then the number of zeros of $f(x)$ is either $\leqq n$ or infinite.

Proof. If $f(x)$ has more than $n$ zeros, then two of them lie in the same conjugacy class by Theorem 2 . By Theorem 4, this class contains infinitely many zeros of $f(x)$.

3. General polynomials. We suppose throughout this section that $[D: K]$ $=d<\infty$. Elements of $K$ are denoted by greek letters. Let $1=e_{1}, \cdots, e_{d}$ be a basis of $D$ over $K$, and let $x=\xi_{1} e_{1}+\cdots+\xi_{d} e_{d}$ be the generic element of $D$. If $f(x)$ is a general polynomial of degree $n$, we can express all its coefficients in terms of the basis $e_{1}, \cdots, e_{d}$. Then after multiplying the factors of each monomial $a_{0} x a_{1} \cdots x a_{k}$ and collecting terms, we obtain

$$
f(x)=f_{1}\left(\xi_{1}, \cdots, \xi_{d}\right) e_{1}+\cdots+f_{d}\left(\xi_{1}, \cdots, \xi_{d}\right) e_{d},
$$

where the $f_{i}\left(\xi_{1}, \cdots, \xi_{d}\right)$ are polynomials in $K\left[\xi_{1}, \cdots, \xi_{d}\right]$. Thus the equation $f(x)=0$ is equivalent to the system $f_{i}\left(\xi_{1}, \cdots, \xi_{d}\right)=0 \quad(i=1, \cdots, d)$. We note that each $f_{i}$ is either identically zero or of degree $\leqq n$.

To avoid endless separation of cases in what follows, we make the convention that 0 is a homogeneous polynomial of degree $n$ for any $n \geqq 0$.

Theorem 6. If $f_{i}\left(\xi_{1}, \cdots, \xi_{d}\right) \in K\left[\xi_{1}, \cdots, \xi_{d}\right] \quad(i=1, \cdots, d)$ are $d$ given polynomials of degree $\leqq n$, then there exists a polynomial $f(x) \in D_{G}[x]$ of degree $\leqq n$ such that $f(x)=\sum_{i=1}^{d} f_{i} e_{i}$.

Proof. The theorem is clearly true if $d=1$, i.e., $D=K$. Assume from now on that $d>1$, so that $D$ is noncommutative. It suffices to show that if the $f_{i}$ are all homogeneous polynomials of degree $n$, then there is a homogeneous polynomial $f(x) \in D_{G}[x]$ of degree $n$ with $f(x)=\sum_{i=1}^{d} f_{i} e_{i}$. (The general case then follows by forming sums.) If $n=0$ the result is obvious. If $n=1$, we have $f_{i}\left(\xi_{1}, \cdots, \xi_{d}\right)=\sum_{j=1}^{d} \alpha_{i j} \xi_{j}$ with $\alpha_{i j} \in K$. Thus the $f_{i}$ define a linear transformation of $D$, considered as a vector space over $K$, into itself. It is our object to show that this transformation is of the form $x \rightarrow f(x)$ for some homogeneous polynomial $f(x) \in D_{G}[x]$ of degree one. Such polynomials have the form $f(x)=\sum a_{v} x b_{v}$, where $a_{v}, b_{v} \in D$. From this it is trivial to verify that the corresponding transformations $x \rightarrow f(x)$ form a ring $R$. We now show that $R$ is doubly transitive. Let $a$ and $b$ be two elements of $D$ which are linearly independent over $K$, and let $c, d$ be any two elements of $D$. Then $a b \neq 0$, and $a b^{-1} \notin K$. Hence there is an element $r \in D$ such that $s=r a b^{-1}-a b^{-1} r \neq 0$. Then $t=b a^{-1} r^{-1}-r^{-1} b a^{-1}$ $\neq 0$. The polynomial

$$
g(x)=\left(r x b^{-1}-x b^{-1} r\right) s^{-1} c+\left(x a^{-1} r^{-1}-r^{-1} x a^{-1}\right) t^{-1} d
$$


satisfies $g(a)=c$ and $g(b)=d$, proving that $R$ is doubly transitive. By a theorem of Jacobson [2, p. 32], $R$ is the ring of all linear transformations of $D$; thus there is an $f(x) \in D_{G}[x]$ such that $f(x)=\sum_{i=1}^{d} f_{i} e_{i}$.

To deal with the case $n>1$ we consider the ring $D_{G}\left[x_{1}, \cdots, x_{n}\right]$ of general polynomials in $n$ indeterminates. A polynomial $p\left(x_{1}, \cdots, x_{n}\right) \in D_{G}\left[x_{1}, \cdots, x_{n}\right]$ is called a multilinear form if it is homogeneous and linear in each indeterminate $x_{k}$. Putting $x_{k}=\sum_{i=1}^{d} \xi_{i}^{(k)} e_{i}$, and expressing the coefficients of $f$ in terms of the basis $e_{1}, \cdots, e_{d}$, we find that

$$
p\left(x_{1}, \cdots, x_{n}\right)=\sum_{i=1}^{d} p_{i}\left(\xi_{1}^{(1)}, \cdots, \xi_{d}^{(n)}\right) e_{i},
$$

where the $p_{i}$ are polynomials in $K\left[\xi_{1}^{(1)}, \cdots, \xi_{d}^{(n)}\right]$. Moreover $p$ is multilinear if and only if all the $p_{i}$ are multilinear (i.e., linear in each set of indeterminates $\left.\xi_{1}^{(k)}, \cdots, \xi_{d}^{(k)}\right)$. We assert that given any $d$ multilinear forms $p_{i}$ $\in K\left[\xi_{1}^{(1)}, \cdots, \xi_{d}^{(n)}\right]$, there exists a multilinear form $p \in D_{G}\left[x_{1}, \cdots, x_{n}\right]$ such that $p=\sum f_{i} e_{i}$. For let

$$
g_{k}\left(\xi_{1}^{(k)}, \cdots, \xi_{d}^{(k)}\right) \in K\left[\xi_{1}^{(k)}, \cdots, \xi_{d}^{(k)}\right] \quad(k=1, \cdots, n)
$$

be given linear forms. By what we have already shown there exist polynomials $h_{k}\left(x_{k}\right) \in D_{G}\left[x_{k}\right] \quad(k=1, \cdots, n)$ such that $h_{1}\left(x_{1}\right)=g_{1} e_{i}$, and $h_{k}\left(x_{k}\right)$ $=g_{k} e_{1}$ for $k>1$. Then (recalling that $e_{1}=1$ ) we have $h_{1}\left(x_{1}\right) \cdots h_{n}\left(x_{n}\right)$ $=g_{1} \cdots g_{n} e_{i}$. The terms of the given polynomial $p_{i}$ are of the form $g_{1} \cdots g_{n}$, so $p_{i}$ is a sum of such polynomials. Hence $p_{i} e_{i}$ is the sum of the corresponding polynomials $h_{1}\left(x_{1}\right) \ldots h_{n}\left(x_{n}\right) \in D_{G}\left[x_{1}, \cdots, x_{n}\right]$. Applying this fact for each $i=1, \cdots, d$ and summing over $i$ we obtain a multilinear form $p\left(x_{1}, \cdots, x_{n}\right) \in D_{G}\left[x_{1}, \cdots, x_{n}\right]$ such that $p=\sum p_{i} e_{i}$.

Now suppose that

$$
f_{i}\left(\xi_{1}, \cdots, \xi_{d}\right) \in K\left[\xi_{1}, \cdots, \xi_{d}\right] \quad(i=1, \cdots, d)
$$

are $d$ given homogeneous polynomials of degree $n$. By "polarization" we construct multilinear polynomials $p_{i}\left(\xi_{1}^{(1)}, \cdots, \xi_{d}^{(n)}\right) \in K\left[\xi_{1}^{(1)}, \cdots, \xi_{d}^{(n)}\right]$ such that $p_{i}$ reduces to $f_{i}$ under the substitution $\xi_{1}^{(1)}=\cdots=\xi_{1}^{(n)}=\xi_{1}, \cdots, \xi_{d}^{(1)}=\cdots$ $=\xi_{d}^{(n)}=\xi_{d}$. By what we have shown, there is a polynomial $p\left(x_{1}, \cdots, x_{n}\right)$ $\in D_{G}\left[x_{1}, \cdots, x_{n}\right]$ such that $p=\sum p_{i} e_{i}$. Then $f(x)=p(x, \cdots, x) \in D_{G}[x]$ satisfies $f=\sum f_{i} e_{i}$, completing the proof.

Theorem 7. Let $K$ be any infinite field, and let $\left\{n_{1}, n_{2}, \cdots, n_{d}\right\}$ be any set of positive integers. Suppose $1 \leqq h \leqq n_{1} n_{2} \cdots n_{d}$. Then there exist $d$ polynomials $f_{i}\left(\xi_{1}, \cdots, \xi_{d}\right) \in K\left[\xi_{1}, \cdots, \xi_{d}\right] \quad(i=1, \cdots, d)$ such that $\operatorname{deg} f_{i}=n_{i}$, and such that the system $f_{i}\left(\xi_{1}, \cdots, \xi_{d}\right)=0 \quad(i=1, \cdots, d)$ has exactly $h$ solutions. The same conclusion holds for $h=0$, provided that $d>1$.

Proof. It is convenient to prove a stronger statement, namely that the $f_{i}$ can be chosen so that $f_{i}$ is a product of $n_{i}$ linear polynomials, and such that if $p_{i}$ is any linear factor of $f_{i}(i=1, \cdots, d)$, then $p_{1}, \cdots, p_{d}$ are linearly 
independent over $K$. Consider first the case $d=1$, and write $n_{1}=n$, $\xi_{1}=\xi$. We have $0 \leqq h \leqq n$. Since $K$ is infinite, there exist $h$ distinct elements $\alpha_{1}, \cdots, \alpha_{h} \in K$. The polynomial $f(\xi)=\left(\xi-\alpha_{1}\right)^{n-h+1}\left(\xi-\alpha_{2}\right) \cdots\left(\xi-\alpha_{h}\right)$ clearly does what is required.

Assume next that $d>1$, and that $n_{1}=n_{2}=\cdots=n_{d}=1$. Then $0 \leqq h$ $\leqq 1$. If $h=1$, set $f_{i}=\xi_{i}$, for all $i$. If $h=0$ put $f_{1}=\xi_{1}, f_{2}=\xi_{1}+1$, and $f_{i}=\xi_{i}$ for all $i>2$. (Note that these polynomials are linearly independent over $K$.) The proof now proceeds by induction on $d$, and for fixed $d$ by induction on $s=\sum_{i=1}^{d} n_{i}$. Assume then that $s>d$, and that the theorem is true for all sets $\left\{m_{1}, \cdots, m_{c}\right\}$, where $c<d$, and also for all sets $\left\{m_{1}, \cdots, m_{d}\right\}$, where $\sum_{i=1}^{d} m_{i}<s$. Suppose without loss of generality that $n_{1}>1$. Then the induction hypothesis can be applied to the set $\left\{n_{1}-1, n_{2}, \cdots, n_{d}\right\}$. Thus for any $h$ in the range $0 \leqq h \leqq\left(n_{1}-1\right) n_{2} \cdots n_{d}$ we can find polynomials $g_{i}\left(\xi_{1}, \cdots, \xi_{d}\right) \quad(i=1, \cdots, d)$ of the special type described above, such that $\operatorname{deg} g_{1}=n_{1}-1, \operatorname{deg} g_{i}=n_{i}$ for $i>1$, and such that the system $g_{i}\left(\xi_{1}, \cdots, \xi_{d}\right)=0$ has exactly $h$ solutions. Let $p\left(\xi_{1}, \cdots, \xi_{d}\right)$ be one of the linear factors of $g_{1}\left(\xi_{1}, \cdots, \xi_{d}\right)$. Then set $f_{1}=p g_{1}$ and $f_{i}=g_{i}$ for $i>1$. Clearly the polynomials $f_{i}$ have the desired property.

We may therefore suppose that $\left(n_{1}-1\right) n_{2} \cdots n_{d}<h \leqq n_{1} n_{2} \cdots n_{d}$. Write $h=\left(n_{1}-1\right) n_{2} \cdots n_{d}+k$, where $1 \leqq k \leqq n_{2} \cdots n_{d}$. By induction there exist polynomials $g_{i} \in K\left[\xi_{2}, \cdots, \xi_{d}\right](i=2, \cdots, d)$ of our special type such that $\operatorname{deg} g_{i}=n_{i}$, and such that the system $g_{i}\left(\xi_{2}, \cdots, \xi_{d}\right)=0 \quad(i=2, \cdots, d)$ has exactly $k$ solutions. Let the decomposition of $g_{i}$ into linear factors be $g_{i}=p_{i}^{(1)} \cdots p_{i}^{\left(n_{i}\right)}(i=2, \cdots, d)$. Set $f_{i}=\prod_{j=1}^{n_{i}}\left(\alpha_{i j} \xi_{1}+p_{i}^{(j)}\right) \quad(i=2, \cdots, d)$, where the $\alpha_{i j}$ are elements in $K$ which will be specified later. Put

$$
f_{1}=\xi_{1} \prod_{j=2}^{n_{1}}\left(\beta_{j} \xi_{1}+p_{1}^{(j)}\right),
$$

where the $\beta_{j}$ are elements of $K$ to be specified later, and the $p_{1}^{())}$are linear polynomials in $\xi_{2}, \cdots, \xi_{d}$, to be determined. There is no nontrivial relation of the form

$$
\lambda_{1} \xi_{1}+\lambda_{2}\left(\alpha_{2 r} \xi_{1}+p_{2}^{(r)}\right)+\cdots+\lambda_{d}\left(\alpha_{d t} \xi_{1}+p_{d}^{(t)}=0 .\right.
$$

For setting $\xi_{1}=0$ we see that $\lambda_{2}=\ldots=\lambda_{d}=0$ by the independence of $p_{2}^{(r)}, \cdots, p_{d}^{(t)}$. Hence $\lambda_{1}=0$. Now choose the polynomials $p_{1}^{(j)}(j>1)$ so that $p_{1}^{(j)}, p_{2}^{(r)}, \cdots, p_{d}^{(t)}$ are linearly independent for all choices of $j, r, \cdots, t$. This can be done since the set $V$ of linear polynomials in $\xi_{2}, \cdots, \xi_{d}$ is a $d$-dimensional vector space over the infinite field $K$, and we need merely avoid a finite number of $(d-1)$-dimensional subspaces of $V$ in choosing the $p_{1}^{(j)}$. Then it is clear that $\beta_{j} \xi_{1}+p_{1}^{(j)}, \alpha_{2 r} \xi_{1}+p_{2}^{(r)}, \ldots, \alpha_{d t} \xi_{1}+p_{d}^{(t)}$ are linearly independent. Furthermore the $d \times(d-1)$ matrix formed by the coefficients of $\xi_{2}, \ldots, \xi_{d}$ in the polynomials $p_{1}^{(j)}, p_{2}^{(r)} \ldots, p_{d}^{(t)}$ has rank $d-1$. Hence 
by avoiding a finite number of proper subspaces in the space $W$ of vectors whose coordinates are the $\beta_{j}$ and $\alpha_{i j}$, we can choose the $\alpha$ 's and $\beta$ 's so that the matrix formed by the coefficients of $\xi_{1}, \ldots, \xi_{d}$ in the polynomials $\beta_{j} \xi_{1}$ $+p_{1}^{())}, \alpha_{2 r} \xi_{1}+p_{2}^{(r)}, \ldots, \alpha_{d t} \xi_{1}+p_{d}^{(t)}$ is nonsingular for all choices of $j>1$, $r, \ldots, t$. Then the system $\beta_{j} \xi_{1}+p_{1}^{(l)}=\alpha_{2 r} \xi_{1}+p_{2}^{(r)}=\ldots=\alpha_{d t} \xi_{1}+p_{d}^{(t)}=0$ has a unique solution for each $j>1, r, \ldots, t$. By avoiding a further finite set of subspaces of $W$, we can insure that no $d+1$ of these equations have a common solution, so that the solutions corresponding to different choices of $j, r, \ldots, t$ are distinct.

Now consider the system $f_{i}\left(\xi_{1}, \cdots, \xi_{d}\right)=0(i=1, \cdots, d)$. For this to be satisfied, some linear factor of each $f_{i}$ must vanish. If $\xi_{1}=0$, then the system reduces to $g_{i}\left(\xi_{2}, \ldots, \xi_{d}\right)=0(i=2, \ldots, d)$. This has $k$ solutions by the construction of the $g_{i}$. If $\xi_{1} \neq 0$, we get exactly one solution for every choice of a linear factor from each of the polynomials $f_{1}, \ldots, f_{d}$. There are $\left(n_{1}-1\right) n_{2} \ldots n_{d}$ such choices, and therefore the total number of solutions is $k+\left(n_{1}-1\right) n_{2} \ldots n_{d}=h$. This completes the proof.

THEOREм 8. Let $D$ be a noncommutative division ring with $[D: K]=d$ $<\infty$. Suppose $n \geqq 1$, and let $h$ be an integer satisfying $0 \leqq h \leqq n^{d}$. Then there is a polynomial $f(x) \in D_{G}[x]$ of degree $n$ with $N(f)=h$.

Proof. By Theorem 7 with $n_{1}=\ldots=n_{d}=n$, we can find $d$ polynomials $f_{i}\left(\xi_{1}, \ldots, \xi_{d}\right) \in K\left[\xi_{1}, \ldots, \xi_{d}\right]$ of degree $n$ such that the system $f_{i}\left(\xi_{1}, \ldots, \xi_{d}\right)=0$ $(i=1, \ldots, d)$ has exactly $h$ solutions. By Theorem 6 there is a polynomial $f(x) \in D_{G}[x]$ of degree $\leqq n$ such that $f(x)=\sum_{i=1}^{d} f_{i} e_{i}$. Clearly $\operatorname{deg} f=n$, and $N(f)=h$.

The question of what values $>n^{d}$, if any, can be assumed by $N(f)$ for polynomials $f(x) \in D_{G}[x]$ of degree $n$, is extremely deep, and depends on the arithmetic nature of $K$. By Bézout's theorem we know that if $n^{d}$ $<N(f)<\infty$, then the system $f_{i}\left(\xi_{1}, \ldots, \xi_{d}\right)=0$ has infinitely many solutions in the algebraic closure $\bar{K}$. But of course $K \neq \bar{K}$, since there are no division rings of finite dimension $d>1$ over an algebraically closed field. Thus we gain little information about the zeros of the system $f_{i}=0$ in $K$.

For example, let $K=\boldsymbol{Q}(\sqrt{ }-3)$, where $\boldsymbol{Q}$ is the rational field. Let $D$ be a division ring with center $K$ such that $[D: K]=4$. Then the polynomial

$$
f(x)=\left(\xi_{3}-\xi_{2}^{2}\right) e_{1}+\left(\xi_{2} \xi_{3}-3 \xi_{1}^{2}-3 \xi_{1}-1\right) e_{2}+\left(\xi_{4}^{2}-1\right) e_{3}
$$

has degree 2 , but has exactly 18 zeros in $D$. To see this, we consider the system

$$
\begin{aligned}
\xi_{3} & =\xi_{2}^{2}, \\
\xi_{2} \xi_{3} & =3 \xi_{1}^{2}+3 \xi_{1}+1, \\
\xi_{4}^{2} & =1 .
\end{aligned}
$$


Eliminating $\xi_{3}$ from the first two equations we obtain

$$
\xi_{2}^{3}=3 \xi_{1}^{2}+3 \xi_{1}+1=\left(\xi_{1}+1\right)^{3}-\xi_{1}^{3} \text {. }
$$

By Fermat's last theorem for cubes, the only solutions in $Q(\sqrt{ }-3)$ are such that $\xi_{2}=0$ or $\xi_{1}+1=0$ or $\xi_{1}=0$. There are nine such solutions. Once $\xi_{1}, \xi_{2}$ are known, $\xi_{3}$ is uniquely determined, and $\xi_{4}= \pm 1$. Hence our system has precisely eighteen solutions in $K$, as asserted. On the other hand, $n^{d}=2^{4}=16$.

\section{References}

1. I. N. Herstein, Conjugates in division rings, Proc. Amer. Math. Soc. 7 (1956), 1021-1022. 2. N. Jacobson, Structure of rings, Amer. Math. Soc. Colloq. Publ. Vol. 37, Amer. Math. Soc., Providence, R. I., 1956.

3. A. R. Richardson, Equations over a division algebra, Messenger of Mathematics 57 (1927), 1-6.

4. H. Rohrbach, Review of paper [3], Jahrbuch über die Fortschritte der Mathematik 53 (1927), 122.

\section{University OF CALIFORNIA,}

Los Angeles, Calufornia 